\title{
Biomedical microdevices: the next phase of highlighting scientific discoveries in the field of micro-nanotechnologies for medicine
}

\author{
Alessandro Grattoni ${ }^{1,2,3} \cdot$ Arum Han $^{4,5}$ \\ Published online: 16 November 2021 \\ (c) The Author(s), under exclusive licence to Springer Science+Business Media, LLC, part of Springer Nature 2021
}

Following decades of research and development, biomedical micro and nanosystems have permeated our lives and have become valuable technologies for healthcare as well as in biotechnology, bioengineering, and life sciences. Miniaturization has allowed to leverage properties of materials and fluids that are at the foundation of diagnostic devices, therapeutics, and preventative systems currently in clinical use. They are also key elements of high-throughput testing devices in the rapidly expanding biotechnology and bioengineering fields. Further, integration of miniaturized systems with living cell systems such as in organ-on-a-chip technologies, has enabled innovation and scientific discoveries. With this wealth of developments, nowadays these technologies seem quite ordinary. However, 25 years ago most of these concepts and applications were at their infancy. It was at that time that a few scientists embraced the challenge to dig deep into the 'small' new world, explore and disseminate new opportunities to the scientific community and industry. Micro- and nano-scale engineering of molecular constructs, materials, and devices emerged as holding promise for breakthrough innovations in nearly every field of science and technology.

Alessandro Grattoni

agrattoni@houstonmethodist.org

$\triangle$ Arum Han

arum.han@ece.tamu.edu

1 Department of Nanomedicine, Houston Methodist Research Institute, Houston, USA

2 Department of Surgery, Houston Methodist Hospital, Houston, USA

3 Department of Radiation Oncology, Houston Methodist Hospital, Houston, USA

4 Department of Electrical and Computer Engineering, Texas A\&M University, Houston, USA

5 Department of Biomedical Engineering, Texas A\&M University, Houston, USA
Among these innovators is Dr. Mauro Ferrari. In the early 90's at the University of California at Berkeley, Dr. Ferrari began investigating fluidic system from microscale to low end of the nanoscale. He applied his knowledge in engineering and materials science with mathematics and studied molecular transport phenomena in fluid confined in such tight spaces. In comparison to the macroscale, he identified differences in fluid transport generated by the dominant role of forces at the fluid-wall interface when fluidic systems approach the size of the fluid molecules. With intuition on the potential of his scientific observations, he engaged in nanofabricating fluidic membranes using silicon semiconductor technologies and applied it to drug delivery, cell transplantation, and molecular sieving.

It was at this time that Dr. Ferrari founded Biomedical Microdevices as a scientific journal dedicated to the dissemination of new scientific discoveries and development in the field of micro- and nanotechnologies applied to medicine, together with other pioneers in the field. Since its inaugural issue in 1998, Biomedical Microdevices grew under Dr. Ferrari's leadership and developed for more than 20 years to become a reference journal in the field, which has contributed to shaping this field. One hundred and six (106) Issues comprising more than 1800 articles have been published so far. Following 23 years of dedicated efforts to the journal, in early $2021 \mathrm{Dr}$. Ferrari stepped down from his position at Biomedical Microdevices, while maintaining an honorary title of Founding Editor-in-Chief and an advisory role.

Since September 2021, we, Dr. Alessandro Grattoni and Dr. Arum Han, have taken up the leadership role as the Editor-inChief for Biomedical Microdevices. By means of an introduction, we provide below our biographical synopses.

Dr. Grattoni is the Frank J. and Jean Raymond Centennial Chair, Chairman and Professor of the Department of Nanomedicine at the Houston Methodist Research Institute (HMRI), and Professor in the Department of Surgery and Department of Radiation Oncology at the Houston Methodist Hospital. His research activities are dedicated to the 
development and clinical translation of implantable nanofluidic platforms for controlled, long-term drug delivery and cell transplantation. He has also engaged in the analysis of electrokinetic transport in nanochannels for modulating the release of therapeutics from reservoirs. This is to achieve a remotely controlled drug delivery implant, which will enable telemedicine approaches and personalized treatment of patients. Current areas of research include long-acting delivery systems for HIV pre-exposure prophylaxis (PrEP), intratumoral radio-immunotherapy, and endocrine diseases. Additionally, Grattoni's laboratory is developing novel 3D-printed encapsulation platform technologies for cell transplantation and therapeutics. Dr. Grattoni established the Center for Space Nanomedicine at HMRI, focused on leveraging microgravity on the International Space Station for the investigation of nanomedicine for applications on Earth and in Space. In this context, he has so far conducted five microgravity investigations on board of the International Space Station.

Dr. Arum Han is a Professor of the Department of Electrical and Computer Engineering, and is the Chancellor's EDGES Fellow and the Presidential Impact Fellow of the Texas A\&M University. He also holds a joint appointment in the Department of Biomedical Engineering, and is a Graduate Faculty of the Texas A\&M Health Science Center, Faculty of the Texas A\&M Institute for Neuroscience, and Faculty of Toxicology. His research focuses on the development of microfluidic, lab-on-a-chip, and organ-on-a-chip systems that enable unique biological experiments at high throughput and high accuracy that can then be readily adopted by the broad bio/medical science community. He has pioneered the area of high-throughput microfluidics for microbiology applications, and have been applying these technologies for synthetic biology, host-pathogen interactions, infectious disease, and microbial bioproduction applications. Developing technologies that enable complex multi-step biological assays that involves mammalian cells, microorganisms, and viruses to be conducted in high-throughput microfluidics format are some of his key accomplishments. $\mathrm{He}$ has also pioneered the area of organ-on-a-chip systems for feto-maternal interface and preterm birth research, and is applying these microphysiological system models for new therapeutic development against preterm birth.

Building on Dr. Ferrari's numerous achievements, we are committed to grow the journal to become the leading publication in the field of micro- and nanotechnologies for medicine and life sciences. We envision Biomedical Microdevices to transform into the main platform for publishing the latest scientific and engineering discoveries, for leading experts in the field to provide their prospective on where the field is moving to, and where rising star researchers can present their cutting-edge advances.

For this, we understand the importance of timely article review, as well as the relevance of social media platforms for broad dissemination of scientific and engineering discoveries. Further, we will welcome and solicit topical collections dedicated to emerging areas in the field, as well as prospective and review articles by leading researchers, while maintaining high standards for quality of publication and ethics in research. In addition, we are committed to providing a diverse and inclusive environment for all researchers, which will be reflected in the editorial team of Biomedical Microdevices.

As we open the second chapter of this journal, we look forward to serving the broad scientific community of this exciting and growing field of science and engineering and working with our dedicated editorial team to promptly publish the most exciting scientific and engineering discoveries.

Publisher's Note Springer Nature remains neutral with regard to jurisdictional claims in published maps and institutional affiliations. 